\title{
Medical education in Korea from a German student's perspective
}

\author{
Richard Krueger
}

Faculty of Medicine, Ludwig-Maximilians-Universität München, München, Germany

\section{Introduction}

I am a 6th year medical student from Ludwig Maximilian University of Munich, Germany. I spent 2 months as an observer at the departments of colorectal, hepatobiliary and pancreatic, gastric and esophageal, breast and endocrine, transplant, gynecological surgery, and obstetrics at Korea University Anam Hospital in Seoul. The purpose of this paper is to share my insights about Korean medical education based on what I experienced during my clinical rotation. Before describing my thoughts and opinions on the strengths and weaknesses of medical education in Korea, I would like to introduce the German medical education system to provide some basis for comparison.

\section{German medical education}

In Germany, admission to medical school is based on the average grade from the last 2 years of high school. Only the best are accepted and offered one of the 9,200 seats at one of the 38 medical faculties in Germany. There is no entrance exam besides a natural sciencebased intelligence test that some universities have introduced in the last decade. Outstanding results in this test can add some valuable points to the high school average grade, which, however, will always be the basis of valuation for the admission to medical school. Each student can take this test only once during their lifetime; there is no second chance. The medical curriculum takes 6 years, and it is divided into three sections:

\section{Two years of preclinical basic sciences}

This includes courses such as biology, physics, chemistry, histology, anatomy, biochemistry, physiology, and psychiatry, and ends with the first part of the medical licensing exam (written and oral), which is generated by experts of a national foundation. Every eligible medical student from every university must take this same exam at the same time. The $10 \%-20 \%$ of the
Received: February 11, 2019 • Revised: February 18, 2019 • Accepted: February 19, 2019 Corresponding Author: Richard Krueger (https://orcid.org/0000-0001-7286-2900) Detmolder Strasse 16, Bielefeld, Germany

Tel: +49.1775063274 email: richard.krueger@campus.lmu.de
Korean J Med Educ 2019 Mar; 31(1): 83-87.

https://doi.org/10.3946/kjme.2019.121

eISSN: 2005-7288

(C) The Korean Society of Medical Education. All rights reserved. This is an open-access article distributed under the terms of the Creative Commons Attribution Non-Commercial License (http:// creativecommons.org/licenses/by-nc/3.0/), which permits unrestricted non-commercial use, distribution, and reproduction in any medium, provided the original work is properly cited. 
examinees who fail get two chances to retake the exam before getting expelled from medical school in Germany. During the summer and winter holidays of the first 2 years, medical students have to complete a 3-month nursing internship to get to know the valuable work of nurses. In these first 2 years, most universities begin to teach clinical examination courses such as how to set an electrocardiogram, how to take a blood sample, and how to auscultate the heart and lungs.

\section{Three years of clinical sciences}

This includes courses such as pediatrics, orthopedics, anesthesiology, and ophthalmology, and ends with the second part of the medical licensing exam (written). The same national foundation provides the nationwide patient case-based questions. The $1.5 \%-2 \%$ of the examinees who fail get two chances to retake the exam before getting exmatriculated from medical school in Germany. In their fifth year, medical students are to complete 4 months of short-term clinical rotations, spending between 3-14 days in different departments. This period comprises seminars and observerships, and it is intended to give students insight into clinical work and subspecialties without giving them any responsibility. During the summer and winter holidays of the third to fifth year, a total of 4 months of medical internships have to be completed by students in family medicine and in- and outpatient departments of choice. During these internships, students stay in one department for a month and are part of the medical team. They have their own responsibilities, including assisting in surgeries taking patients' anamnesis during admission, taking blood samples, and performing examinations. Throughout this period, students learn to deal with patients and to work in a team. Professors and supervisors encourage students to give their opinion and answer students' questions about operation techniques, treatment, and prescriptions. Afterwards, both sides, the students and the doctors, evaluate each other for optimized teaching.

\section{One practical year}

This is divided into three full-time clinical rotations, each lasting about 4 months, and ends with the third part of the medical licensing exam (oral). This last exam is provided by the student's university, and comprises clinical questions about the three different departments that the student attended in year six and one randomly assigned department. This last exam evaluates the student's clinical knowledge; it is not intended to fail students. This explains this third medical licensing exam's low failure rate of $0.5 \%$. In this final year, students are included in the daily work with patients at the ward, and they assist in surgeries. They have more responsibilities and patient contact than junior students have and are also included in the medical personnel planning. Additionally, students are expected to work independently from doctors although they are supervised. Besides teaching and weekly seminars, students work in small groups of four to six, focusing on special clinical cases that are adapted according to their wishes and interests. The theoretical university education is officially finished after year five. In the sixth and last year, students learn to work and to think like a resident doctor and hone practical skills such as holding the camera during laparoscopic surgeries and finishing the surgery with sub- and cutaneous stitches. In the last year, rotations in internal medicine and surgery are mandatory. One elective rotation can be freely chosen from all the clinical specialties. One of the three rotations can be equally split in two. Therefore, I was able to spend 2 months at the Korea University College of Medicine.

In general, we can say the longer you study, the lower are your chances of failing an exam and the higher your 
medical responsibility toward your patients and colleagues. Theoretically, every rotation/internship of the German medical curriculum can be undertaken at any university hospital or related clinic in Germany or worldwide. About 30\%-50\% of Germany's medical students go abroad for internships or as visiting students during their studies. The European Union developed a student exchange system by providing an international accreditation system for academic performances abroad and non-bureaucratic scholarships for every student, called Erasmus+.

After graduation from medical school, young German doctors start their residency in the department of choice, where he/she would like to specialize. The residency usually lasts 5 years. There are no rotations or other internships outside of the chosen specialty. This is because students have already acquired practical skills during their last year of studies and must immediately start their residency with the team they will probably work with for several years. Residents are responsible for the admission of patients, the work at the ward, basic examinations, and are to assist senior doctors in surgeries. At the daily morning meeting, they are asked by their supervisors to provide their proposal for treatment and medication. During the ward rounds, they present their patients to the senior doctor and function as the first point of contact for the patient and the supervisor. Then senior doctors make the final decision for the treatment together with the patient and the resident. During the night shift, resident doctors are responsible for handling emergencies. In case of inconveniences or problems, they can ask their supervisor, who would be on standby at the hospital, for help.

\section{Personal thoughts on Korean medical education}

The first 2 years of medical studies in Korea is called 'pre-medical,' which differs significantly from all the European medical education systems. Most of Europe's medical education is 5 or 6 years long and does not have this division of pre-medical and medical studies; medical studies start from the very first year. During these first 2 years of medical education in Korea, students can choose from humanistic subjects such as philosophy or languages and follow some medicine-related subjects like biology. The Korean students I met and interacted with opined that the most difficult period for them is the third year of medical studies, which is overloaded with the contents that German medical students complete in their first 2 years. To put it concisely, Korean medical students complete a large portion of their medical curriculum during year three to year six, whereas Germany's medical curriculum is more evenly spread throughout the 6 years.

Due to this, my Korean colleagues have very intense schedules, easily reaching 12-14 hours of study time per day. Furthermore, their medical education is much more focused on theoretical rather than practical education and bedside teaching. I was very surprised to learn that Korean students would have very detailed knowledge on tumor classifications or millimeter resection margins but often have difficulties with general medical comprehension.

Example: Students could answer what the classification IIIb of a Klatskin tumor is but would not understand how a right portal vein embolization would lead to hypertrophy of the left hepatic lobe. They know rare labor-inducing medicament side effects but do not know the general function of the labia minora of the female 
genital is. Students would rather remain silent if they do not know the exact answer to a question than give an education guess, and thus, an insight into their way of thinking.

I was also astonished by the strong dominance of hierarchy and the fear of losing face in front of colleagues at the hospital. On the one hand, Korean students barely pose questions to a senior doctor or professor. By misinterpreting their silence in the operation room and taking it for complete understanding, I often asked Korean students questions about the operation we were watching. They usually did not know the answers and might have asked the same questions themselves, but would not ask because it might be seen as ignorance. They were also afraid of being asked a question in return and not knowing the answer. On the other hand, on the rare occasions that a professor addressed the students, I often recognized he/she would ask for very precise information about the patient's anamnesis or medicament side-effects instead of supporting their medical thinking with questions like: "What do you know about breast cancer?" or "What do you think is a good approach for a nephrectomy?"

However, the Korean medical education system also has great advantages:

(1) In Germany, medical students are known to have poor general education because they never studied anything else besides medicine. Korea emphasizes humanistic and general education for all its students by giving them the chance to study social sciences in their first 2 years at university.

(2) In Germany, the personal relation between doctors is very much limited to their professional life. I never witnessed a German team of doctors go out for dinner during the week and even invite students to join them. I think it is a great attitude to share both professional and private life with colleagues, who are the people a doctor would see most time of his/her week.

(3) The clinical rotations for Korean fifth-year medical students last for one and a half years. Although they have less practical teaching in hospital, they experience the daily work of subspecialties and departments much more than German students. I was amazed to have had the opportunity to rotate on a weekly basis from operation room to another and hence to see various highly skilled operations. During internships, German students spend weeks or months in the same department, which does not allow them to experience diverse medical applications in every sub-department like in Korea.

(4) Korea certainly outpaces European countries in medical technology. For example, in Korea University College of Medicine, there are virtual dissections of human bodies, modern broadcasting systems for lectures that are available for students $24 / 7$ online, and international student researchers' conferences that welcome young scientists from several Asian countries. Available technology in Korea University Anam Hospital include online appointment service for medical consultation for patients, routine extensive imagery for more complex medical cases, three-dimensional laparoscopic operations, and highly specialized robotic surgery in every surgery department.

I am grateful for the amazing, open, and friendly attitude shown towards me by each person I met at Korea University Anam Hospital and the Korea University College of Medicine in Seoul. I am thankful for and astounded by the wholehearted welcome and care I experienced. The organization of the clinical rotation and opportunity to participate in any conference, operation, meeting, ward round, lunch, workshop, and presentation was outstanding. I feel honored and glad that I made friends $10.000 \mathrm{~km}$ away from my home country. I am hopeful that I can contribute to the exchange of opinions, experiences, and people between 
our medical faculties, cultures, and countries. I remain deeply grateful for the friendship. Best wishes from Germany!

\section{Summary}

I am convinced that Korea has a high-quality health system and is very successful in its ambitious approach to connect with other leading countries in medical care and research. However, regarding medical education, a more open environment and working relationship between students and doctors can provide easier and more effective communication without a loss of mutual respect. I believe that it is useful for patients, doctors, and students to focus medical student' s education on bedside and practical teaching early on, to increase their understanding and motivation to become good clinicians.

\section{ORCID:}

Richard Krueger: https://orcid.org/0000-0001-7286-2900 Acknowledgements: This essay was written during a 2-month observership at Korea University Anam Hospital of Korea University College of Medicine in Seoul under the guidance of Dr. Young-Mee Lee.

Funding: None.

Conflicts of interest: No potential conflict of interest relevant to this article was reported.

Author contributions: Whole process, ranging from conception of the work to critical revision, was done by the author. 